\title{
Detecting Overlapping Communities in Social Networks by Game Theory and Structural Equivalence Concept
}

\author{
Hamidreza Alvari, Sattar Hashemi, and Ali Hamzeh \\ Department of Electrical and Computer Engineering, Shiraz University, \\ Shiraz, Iran \\ alvari@cse.shirazu.ac.ir, s_hashemi@shirazu.ac.ir, \\ ali@cse.shirazu.ac.ir
}

\begin{abstract}
Most complex networks demonstrate a significant property 'community structure', meaning that the network nodes are often joined together in tightly knit groups or communities, while there are only looser connections between them. Detecting these groups is of great importance and has immediate applications, especially in the popular online social networks like Facebook and Twitter. Many of these networks are divided into overlapping communities, i.e. communities with nodes belonging to more than one community simultaneously. Unfortunately most of the works cannot detect such communities. In this paper, we consider the formation of communities in social networks as an iterative game in a multiagent environment, in which, each node is regarded as an agent trying to be in the communities with members structurally equivalent to her. Remarkable results on the real world and benchmark graphs show efficiency of our approach in detecting overlapping communities compared to the other similar methods.
\end{abstract}

Keywords: Social networks, community detection, overlapping communities, game theory, structural equivalence.

\section{Introduction}

The solution to the Königsberg bridges puzzle in 1736 by Leonhard Euler [1] was perhaps the origin of the graph theory. Since $20^{\text {th }}$ century, graph has been extremely used as a representation tool, for a wide range of complex systems in different areas. Nowadays, graphs and their analysis have become a prominent component in studying such systems and their properties, including social, biological, technological, and informational networks. Emergence of huge amount of data and computational resources provided by the computer revolution has made the processing and analysis of data very hard. Recent rapid expansion of real networks to millions or even billions of vertices has produced a deep change in the way graphs are approached [2-4]. These large networks exhibit interesting common properties, such as high network transitivity [5], power-law degree distributions [6], the existence of repeated local motifs [7] and the outstanding property 'community structure' or 'clustering', 


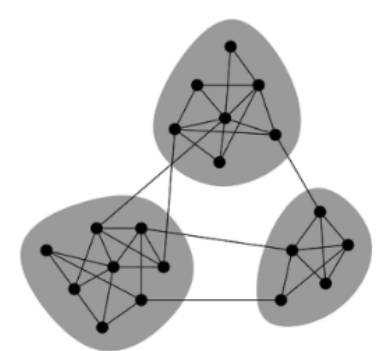

Fig. 1. Shaded sets of vertices show communities of the network, within which there are many edges, with only a smaller number of edges between vertices of different groups [8]

meaning the advent of dense connected groups, modules, clusters or communities of vertices in the graph, and sparser connections between them. The schematic example of a graph with communities is shown in Figure 1.

Because of its high applicability, this property has attracted a lot of researchers from different fields of study. From the important applications of detecting such groups, one can refer to detecting groups within World Wide Web which might correspond to web pages on the related topics [9], groups in social networks like Facebook and Twitter, which often show knit relationships between their members [10], and even groups in a metabolic network which might represent cycles and other functional groupings [11]. In addition, clustering Web clients having similar interests and being geographically near to each other, can improve the performance of services provided on the World Wide Web [12] and Detecting clusters of customers with similar interests in the network of purchase relationships between customers and products of online retailers (e.g. www.amazon.com) can lead to set up efficient recommendation systems and improve business opportunities [13]. As another application, clustering large graphs can help in creating data structures to store the graph more efficiently [14].

Perhaps, one of the most appealing issues which deserves initiating new trend of researches is to address overlapping communities, say, the case in which some nodes in the network belong to more than one community at the same time. An intuitive example of this concept is the membership of each of us in the communities like family, friends, co-workers, etc. Unfortunately only some of the proposed methods (See for example [18-22]) are capable to find these overlapping communities in a given networks.

In this paper, we have proposed a new framework based on game theory and structural equivalence concept to identify the overlapping communities in the networks. To do so, two alternative approaches, named PSGAME and NGGAME, are designed and developed. The former approach incorporates Pearson correlation into game theory, whereas, the later approach makes use of neighborhood relation as similarity measure in the game of concern. In addition, a new similarity measure, neighborhood similarity, established on the neighborhood and adjacency relationships is introduced. This 
measure, calculates similarities between graph vertices. Experiments show that these methods give more accurate results than the other state of art rival approaches.

The measures taken in the rest of the paper are organized as follows. In Section 2 the community detection problem is defined and a brief review of the history of existing methods on it is given in the section 3 . In Section 4, our proposed framework is explained in details and in Section 5 the comparisons between our results and the results of some other popular methods in the literature are mentioned. Finally in Section 6 , the conclusions and hints for future works are discussed.

\section{Problem Statement}

Given an underlying graph $G$, with $|G|=n$ vertices (nodes) and $m$ edges, the community detection problem is to cluster these vertices into $C$ communities or groups based on some predefined measures such that these communities can have intersections (i.e. overlapping communities) or not. However the community detection problem, intuitive at first sight, is in fact, not well defined, because main elements of it (e.g. the community concept) are not precisely defined and there are often some ambiguities in their definitions. This has resulted in presenting so many interpretations of it in the literature.

The main issue on community detection problem is to look for some quantitative measure and definition of community. Unfortunately no universally accepted definition is in hand, because this definition often depends on the underlying problem and its application. But intuitively we expect more edges inside the community compared to the outside of it and the rest of the graph. This simple concept is the basis of the most definitions of community. However social network analysts have distinguished three main classes of definitions for community: local, global and vertex similarity based. According to the local definition, a community is being evaluated independently of the graph, while in the global definition communities are defined with respect to the graph as a whole. Definitions based on the vertex similarity, assume that communities are groups of vertices most similar to each other.

\section{Related Work}

Research on the community detection problem has a long history and has been appeared in several forms and applications including sociology and computer science. The first analysis of community structure in the networks dates back to 1927 and the paper written by Stuart Rice, who looked for clusters of people in small political bodies, based on the similarity of their voting patterns [15]. Since then, many works have been done, which can be categorized into two main groups: optimization methods which look for optimizing some measures and methods with no optimization, which search for some predetermined structures.

From these works, one can refer to the works done by Girvan and Newman in 2002 and 2004 that introduced two important concepts 'edge betweenness' [10] and 'modularity' [16], the work done by Brandes and Erlebach which intoroduced the term 'conductance' [17] and the work done by Palla et.al [20]. Recently some works 
have been done considering overlapping concept. Gregory proposed CONGA [18] and CONGO [19] based on 'split betweenness' to detect overlapping communities in the networks. In 2005, Palla et al. showed that searching for some predetermined structures like fully connected graphs in the network can lead to detecting such communities. Meanwhile very few works are done based on the game theory [21, 23]. These works address the problem of community detection by a game-theoretic framework, in which nodes of a graph are considered as rational agents who want to maximize their payoffs according to some criterion. The game-theoretic methods are grounded with a systematic theory for formation of communities in the networks, as in the real world, which communities are formed based on some purposes, not for optimizing some local or global objectives.

\section{Proposed Framework}

It is naturally plausible if the problem of community detection in the network is regarded as a play of interactions between humans in the society, where each node of the underlying network is considered as a selfish agent, who wants to maximize her total utility. A little concentration on the problem shows that we as humans always need to make deep social relationships with our friends in the society we belong to, or sometimes need to leave them according to our profits. Based on this intrinsic fact, we often choose between join, leave and switch operations in our social interactions. When we think that joining to a community will be good for us because of some reasons, we will join it. When belonging to one community is pernicious for us, we will leave it. On the other hands when we find another community, more profitable, we leave the community we belong to, in order to join to this community, i.e. we switch from one community to another.

Communities are often defined as the groups of the most similar vertices to each other. Therefore one can compute the similarities between each pair of vertices of the network with respect to some local or global property; no matter whether they are connected directly or not. There are a lot of similarity measures in the literature that are at the basis of traditional methods like hierarchical, partitional and spectral clustering [24]. These measures are mainly divided into two categories: when it is possible to embed the graph vertices in a Euclidean space, the most prominent measures are Euclidean distance, Manhattan distance and cosine similarity, but when a graph can't be embedded in space, adjacency relationships between vertices are used (See for example [25]).

In this work we've used two similarity measures based on adjacency relationships and structural equivalence [26]. Two vertices are called structural equivalent if they have the same neighbors, even if they are not directly connected. The measures we used are Pearson correlation and our novel similarity measure, neighborhood similarity. Here, we formulate the community detection problem as an iterative game, in which each node is a rational agent who plays the community formation game hopefully to maximize her total utility based on structural equivalence (similarity). This game will continue until no agent can improve hers. Considering that the underlying network is given in the form of a graph, our framework is as follows. 
Each node of the graph $G$ is considered as an agent that has a personal utility function. Before starting the game, similarities between all agents are calculated by a similarity measure. When starting the game, each agent based on this similarity as her utility, will choose between joining to a new community or existing one, leaving from existing community and switching from existing community to another existing one. A node can also do nothing if these operations don't change her utility. Overlapping community structure of the network will come into the view after agents reach local equilibrium. We used local Nash equilibrium in this game, because reaching global one is not feasible [27].

Naturally when joining to a new community, each of us will be beneficiary, but on the other hands, we must pay some costs (e.g. fees). Therefore, we've used a gain function $Q$ and a loss function $L$ and a utility function $U$, as their difference for each node:

$$
\begin{aligned}
Q_{i}(S) & =\frac{1}{2 m} \sum_{\substack{j=1 \\
j \neq i}}^{n} C_{i j} \delta_{i j} \\
L_{i} & =\frac{1}{m}\left(\left|s_{i}\right|-1\right)
\end{aligned}
$$

In above equations, $S$ indicates the strategy profile of all agents, i.e. labels of memberships of all agents, $m$ is the number of graph edges, $n$ is the number of nodes or agents, $\mathrm{C}_{\mathrm{ij}}$ is the similarity between node $i$ and node $j$, The output of $\delta$ function is 0 or 1 ; when two agents have common labels, i.e. $\left|s_{i} \cap s_{j}\right| \geq 1$, the output is 1 , otherwise 0 . Finally $\left|s_{i}\right|$ is the number of labels agent $i$ belongs to.

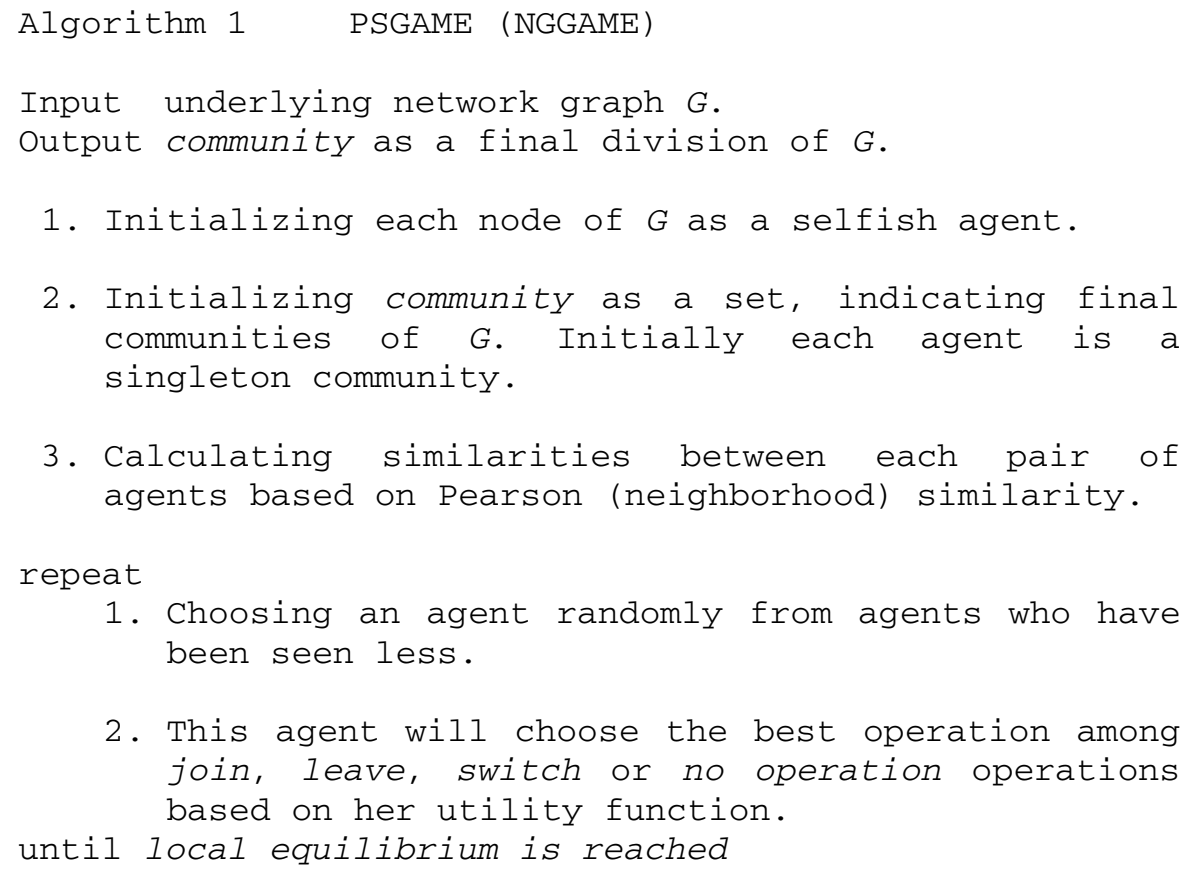


We used this framework in two methods PSGAME and NGGAME, based on whether $C_{i j}$ is calculated by Pearson correlation measure or by neighborhood similarity measure. Pseudocode of our proposed framework is shown in below.

\subsection{PSGAME}

Pearson correlation is a measure related to structural equivalence which calculates correlation between columns or rows of an adjacency matrix. This measure can be used in order to calculate similarities between nodes of a graph. PSGAME is a game based method in which similarity between agents $i$ and $j, \mathrm{C}_{\mathrm{ij}}$, is calculated by Pearson correlation. Here $A$ is an adjacency matrix of $G, \mu_{i}$ is an average and $\sigma_{i}$ is a variance:

$$
\begin{gathered}
C_{i j}=\frac{1}{n \sigma_{i} \sigma_{j}} \sum_{k}\left(A_{i k}-\mu_{i}\right)\left(A_{j k}-\mu_{j}\right) \\
\mu_{i}=\frac{1}{n} \sum_{j} A_{i j} \\
\sigma_{i}=\sqrt{\frac{1}{n} \sum_{j}\left(A_{i j}-\mu_{i}\right)^{2}}
\end{gathered}
$$

In fact, this metric quantifies how similar are two nodes and is measured from -1 to +1 . Score 1 indicates that they are perfectly similar and a score of -1 means that they are dissimilar. In other words, the Pearson correlation score, quantifies how well two data objects fit a line.

\subsection{NGGAME}

Two nodes are most likely to be in one community, if they have common neighbors and are connected directly by an edge. Therefore we proposed a new similarity measure, neighborhood similarity, based on the number of common neighbors, adjacency matrix of the graph and degrees of the nodes. This similarity measurement is in the range $[-1,+1]$. NGGAME is a game based method that uses neighborhood similarity to calculate similarity $C_{i j}$ between agents $i$ and $j$. Let $\omega_{i j}$ be $|\Gamma(i) \cap \Gamma(j)|$ and $\Gamma(i)$ indicates the set of neighbors of node $i$. Assume $A$ is adjacency matrix of $G$, $m$ is the number of edges, $n$ is the number of vertices and $d_{i}$ is the degree of node $i$.

$$
C_{i j}=\left\{\begin{array}{ccc}
\left\{\begin{array}{cc}
\omega_{i j}\left(1-\frac{d_{i} d_{j}}{2 m}\right) & A_{i j}=1 \\
\frac{\omega_{i j}}{n} & A_{i j}=0
\end{array}\right. & \omega_{i j} \geq 1 \\
\left\{\begin{array}{lll}
\frac{d_{i} d_{j}}{4 m} & A_{i j}=1 \\
-\frac{d_{i} d_{j}}{4 m} & A_{i j}=0 &
\end{array} \omega_{i j}=0\right.
\end{array}\right.
$$

When nodes $i$ and $j$ have common neighbors, i.e. $\omega_{i j} \geq 1$ and they are themselves connected directly, i.e. $A_{i j}=1, C_{i j}$ gets its largest value, because it seems that these nodes are very similar. As long as they have no common neighbors and they are 


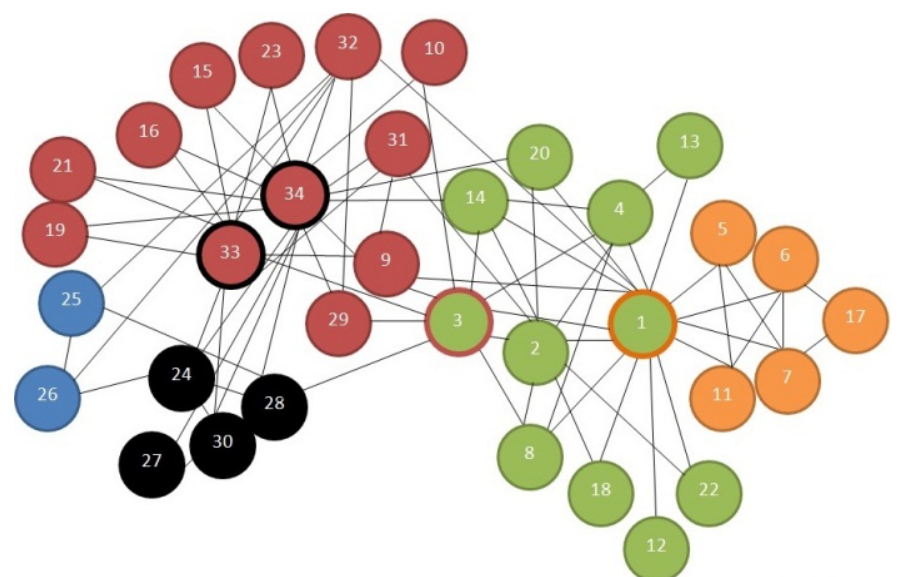

Fig. 2. Communities found by NGGAME on the Zachary network. Nodes with thick colored margins show overlapping communities.

not connected themselves, $C_{i j}$ gets its worst value. These cases and two other cases are shown in above formula.

\section{Experiments}

We conducted our experiments on both real world and benchmark graphs. To evaluate our work, from different metrics used in literature [24], we used recent version of normalized mutual information [28] that can support overlapping communities.

\subsection{Real World Graphs}

We have evaluated our work on real world graphs, Dolphin network [29] and Zachary Karate Club network [30], two well-known networks in the literature with known community structure.

In Dolphin network, the network of bottlenose dolphins living in Doubtful Sound (New Zealand) analyzed by biologist David Lusseau, there are 62 dolphins and edges were set between animals that were seen together more often than expected by chance. On the other hands, Zachary network consists of 34 vertices, the members of a karate club in the United States, who were observed during a period of three years. Edges connect individuals who were observed to interact outside the activities of the club. At some point, a conflict between the club president and the instructor led to the fission of the club in two separate groups, supporting the instructor and the president, respectively [24]. The communities of the Zachary network found by NGGAME method is shown in Figure 2.

\subsection{Benchmark Graphs}

In addition we ran our method on a set of benchmark graphs recently proposed by Lancichinetti and Fortunato [31]. Briefly speaking, the graphs are constructed in the 
following steps: (1) generate the number of communities that each node will belong to; assign the degrees to the nodes based on a power law distribution with exponent $\tau_{1}$; (2) assign the community sizes from another power law distribution with exponent $\tau_{2}$ for a fixed number of communities; (3) generate the bipartite graph between the nodes and the communities with the configuration model; (4) for each node, assign the cross-community degree and internal degrees within each community based on $\mu$ (mixing parameter); (5) build the graphs for each community and the crosscommunity edges with the configuration model.

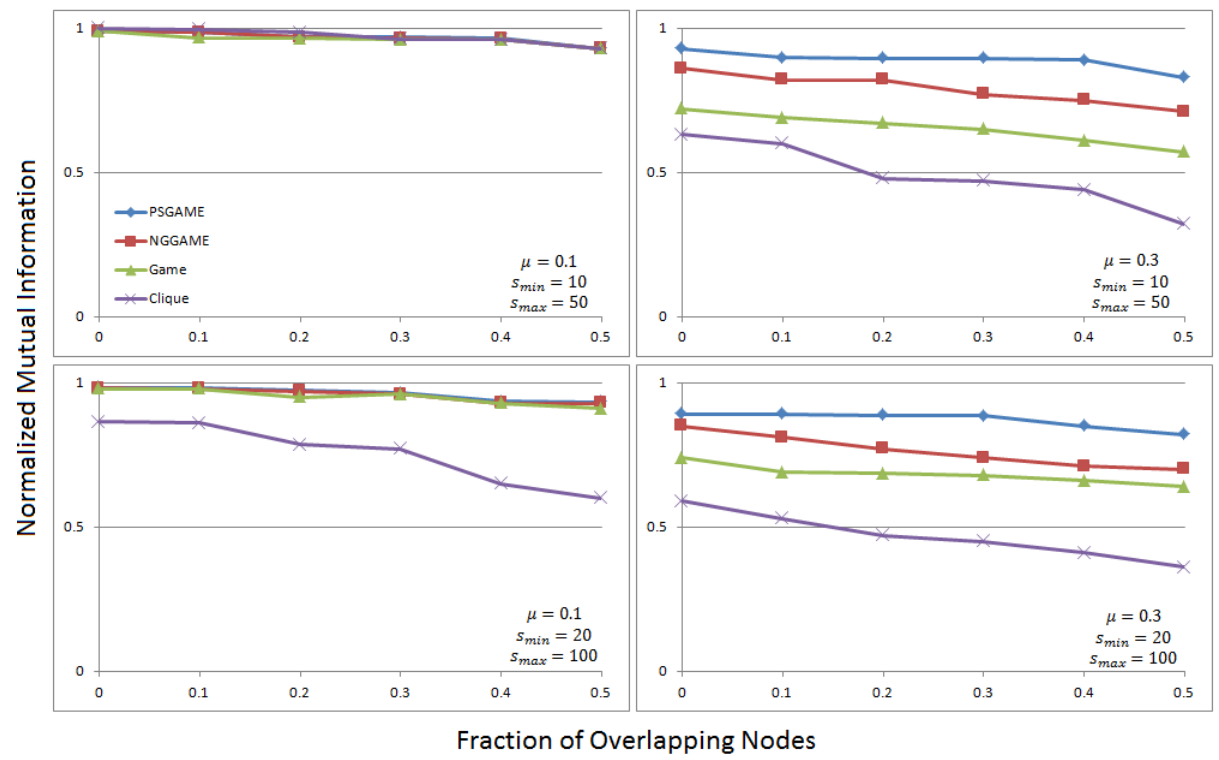

Fig. 3. Comparison between results on the benchmarks with 1000 nodes

The comparative evaluation between different algorithms are shown in Figure 3 where the $y$ axis is the normalized mutual information (NMI) and $x$ axis shows different fractions of overlapping nodes of benchmarks from 0 to 0.5 . The numbers $s_{\min }$ and $s_{\max }$ refer to the limits of communities sizes. We set the other parameters of the benchmarks as $\tau_{1}=2, \tau_{2}=1, k_{\text {avg }}=20, k_{\max }=50$, om $=2$ and two numbers 0.1 and 0.3 for $\mu$ or mixing parameter (i.e. proportion of crossing edges). Results on the benchmarks demonstrate that our approach is superior with respect to the methods that can detect overlapping communities, such as Clique [20] and Game [21]. It is noticeable that Clique algorithm needs the size of cliques as input. In this work we examined clique sizes of 3 to 8 .

Finally as a toy example, a very simple synthetic graph and its communities found by NGGAME and Game are depicted in Figure 4. This example shows that NGGAME can give more reasonable division of underlying graph compared to the Game method. 


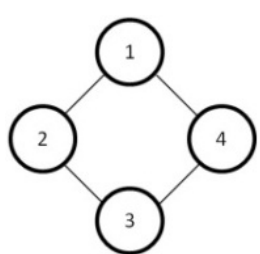

(a)

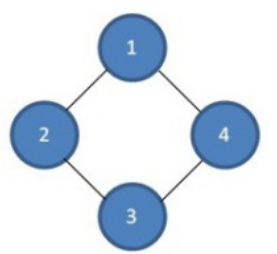

(b)

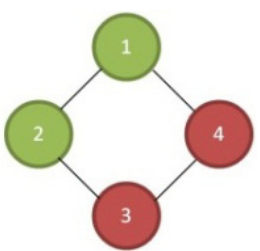

(c)

Fig. 4. (a) A toy (synthetic) example. Communities found by (b) NGGAME, (c) Game

\section{Conclusion and Future Work}

In this work, inspired from social life, we presented a novel framework for detecting overlapping communities in social networks, based on game theory and structural equivalence (similarity) concept. Furthermore we proposed a new similarity measure, neighborhood similarity measure which calculates similarity of graph nodes based on neighborhood relations.

We used this framework in two methods, PSGAME and NGGAME. The first method embeds Pearson correlation measure while the other one uses neighborhood similarity as similarity measure. The motivation behind these methods is that nodes in one community are obviously among the most similar ones to each other compared to the other nodes outside this community. Therefore if we consider each node as a selfish agent who plays the game in order to maximize her sheer goal, this goal will be joining to the most similar nodes to her.

Results show that we have reached to more reasonable divisions of underlying graphs compared to the other existing methods. From two proposed methods, PSGAME presents better results on very large graphs, while NGGAME has reasonable results on the small graphs.

For future works, one can provide another similarity measure or use another existing one instead of Pearson in order to use in this framework. Similarly, loss function used here, can be replaced by a new one. On the other hands, one can embed joint decision concept into this framework. This may have better results at the expense of the high running time. Finally, an extension of this framework to the weighted and directed graphs is straightforward.

\section{References}

1. Euler, L.: Commentarii Academiae Petropolitanae 8, 128 (1736)

2. Albert, R., Barabási, A.-L.: Rev. Mod. Phys. 74(1), 47 (2002)

3. Newman, M.E.J.: SIAM Rev. 45(2), 167 (2003) 
4. Barrat, A., Barthélémy, M., Vespignani, A.: Dynamical processes on complex networks. Cambridge University Press, Cambridge (2008)

5. Watts, D.J., Strogatz, S.H.: Nature. 393, 440-442 (1998)

6. Barabási, A.-L., Albert, R.: Science 286, 509-512 (1999)

7. Milo, R., Shen-Orr, S., Itzkovitz, S., Kashtan, N., Chklovskii, D., Alon, U.: Science 298, 824-827 (2002)

8. Newman, M.E.J.: Proc. Natl. Acad. Sci. USA 103, 8577-8582 (2006)

9. Flake, G.W., Lawrence, S.R., Giles, C.L., Coetzee, F.M.: IEEE Computer 35, 66-71 (2002)

10. Girvan, M., Newman, M.E.J.: Proc. Natl. Acad. Sci. USA 99, 7821-7826 (2002)

11. Chen, J., Yuan, B.: Bioinformatics 22(18), 2283 (2006)

12. Krishnamurthy, B., Wang, J.: SIGCOMM Comput. Commun. Rev. 30(4), 97 (2000)

13. Reddy, P.K., Kitsuregawa, M., Sreekanth, P., Rao, S.S.: A Graph Based Approach to Extract a Neighborhood Customer Community for Collaborative Filtering. In: Bhalla, S. (ed.) DNIS 2002. LNCS, vol. 2544, pp. 188-200. Springer, Heidelberg (2002)

14. Wu, A.Y., Garland, M., Han, J.: In: KDD 2004: Proceedings of the Tenth ACM SIGKDD international conference on Knowledge Discovery and Data Mining, pp. 719-724. ACM Press, New York, NY, USA (2004)

15. Rice, S.A.: Am. Polit. Sci. Rev. 21, 619 (1927)

16. Newman, M.E.J., Girvan, M.: Phys. Rev. E 69(2), 26113 (2004)

17. Brandes, U., Erlebach, T.: Network analysis: methodological foundations. Springer, Berlin (2005)

18. Gregory, S.: An Algorithm to Find Overlapping Community Structure in Networks. In: Kok, J.N., Koronacki, J., Lopez de Mantaras, R., Matwin, S., Mladenič, D., Skowron, A. (eds.) PKDD 2007. LNCS (LNAI), vol. 4702, pp. 91-102. Springer, Heidelberg (2007)

19. Gregory, S.: A Fast Algorithm to Find Overlapping Communities in Networks. In: Daelemans, W., Goethals, B., Morik, K. (eds.) ECML PKDD 2008, Part I. LNCS (LNAI), vol. 5211, pp. 408-423. Springer, Heidelberg (2008)

20. Palla, G., Derényi, I., Farkas, I., Vicsek, T.: Uncovering the Overlapping Community Structure of Complex Networks in Nature and Society. Nature 435, 814-818 (2005)

21. Chen, W., Liu, Z., Sun, X., Wang, Y.: A game-theoretic framework to identify overlapping communities in social networks. Data Min. Knowl. Disc. 21, 224-240 (2010)

22. Zhang, S., Wang, R., Zhang, X.: Identification of Overlapping Community Structure in Complex Networks Using Fuzzy C-means Clustering. Physica A: Statistical Mechanics and its Applications 374(1), 483-490 (2007)

23. Adjeroh, D., Kandaswamy, U.: Game-Theoretic Analysis of Network Community Structure 3(4), 313-325 (2007), doi:10.5019/j.ijcir.2007.112

24. Fortunato, S.: Community detection in graphs. arXiv:0906.0612 (2009)

25. Wasserman, S., Faust, K.: Social Network Analysis: Methods and applications. Cambridge University Press, Cambridge (1994)

26. Lorrain, F., White, H.: J. Math. Social 1, 49 (1971)

27. Aiós-Ferrer, C., Ania, A.: Local equilibria in economic games. Econ. Lett. 70(2), 165-173 (2001)

28. Lancichinetti, A., et al.: Detecting the overlapping and hierarchical community structure in complex networks. Andrea, New J. Phys. 11, 33015 (2009) 
29. Lusseau, D.: The emergent properties of a dolphin social network. Proc. Bio.1. Sci. 270, S186-S188 (2003)

30. Zachary, W.W.: An information flow model for conflict and fission in small groups. J. Anthropol Res. 33(4), 452-473 (1977)

31. Lancichinetti, A., Fortunato, S.: Benchmarks for testing community detection algorithms on directed and weighted graphs with overlapping communities. Phys. Rev. E 80(1), 16118 (2009) 